\title{
Osmophoresis in a dilute suspension of spherical vesicles
}

\author{
Huan J. Keh*, Hung J. Tu \\ Department of Chemical Engineering, National Taiwan University, Taipei, 106-17, Taiwan, ROC
}

Received 19 August 1998; received in revised form 24 December 1998

\begin{abstract}
The osmophoretic motion of two spherical vesicles in a constant gradient of solute concentration is analyzed using a method of reflections. The vesicles are oriented arbitrarily with respect to the gradient, and they are allowed to have different semipermeable membranes, hold arbitrary solutes, and possess unequal sizes. The method of reflections is based on an analysis of the solute concentration and fluid velocity disturbances produced by a single spherical vesicle placed in an arbitrarily varying concentration field. The quasisteady results for two-vesicle interactions are correct to $O\left(r_{12}^{-7}\right)$, where $r_{12}$ is the distance between the vesicle centers. Our analytical results are found to be in good agreement with the numerical solution obtained using spherical bipolar coordinates. Based on a microscopic model the results for two-vesicle interactions are used to determine the effect of the volume fraction of vesicles of each type on the average osmophoretic velocity in a bounded suspension. For a suspension of identical vesicles, this average velocity is increased as the vesicle volume fraction is increased. (C) 1999 Elsevier Science Ltd. All rights reserved.
\end{abstract}

Keywords: Osmophoresis; Semipermeable vesicle; Particle interaction; Effect of volume fraction; Method of reflections; Chemotaxis

\section{Introduction}

All biological membranes, and many synthetic ones, are to some extent semipermeable; that is, they permit the passage of solvent and other small molecular species, but are impermeable to all solutes whose molecules exceed a certain size. Thus, when two solutions of such larger molecules are separated by a semipermeable membrane, with a higher concentration of solute

\footnotetext{
* Corresponding author.

E-mail address: huan@ccms.ntu.edu.tw (H.J. Keh).
}

0301-9322/00/\$ - see front matter (C) 1999 Elsevier Science Ltd. All rights reserved.

PII: S0301-9322(99)00002-6 
on one side than on the other, the fact that the chemical potentials on the two sides will tend to equilibrium causes solvent to flow across the membrane toward the side with higher solute concentration. This is the phenomenon of osmosis; it still occurs to a certain extent when the solute molecules can cross the membrane but experience more resistance in doing so than the solvent molecules. The osmotic flow of solvent can be prevented by applying a pressure to the solution of higher concentration which is greater than the pressure on the solution at the other side by an amount equal to $\sigma \Delta \Pi$, where $\Delta \Pi$ is the difference in osmotic pressure between the two solutions and $\sigma$ is a reflection coefficient characterizing the degree to which the solute molecules are rejected from the membrane. For a semipermeable membrane, $\sigma=1$; for a nonselective membrane, $\sigma=0$. The osmotic pressure $\Pi$ is linearly related to the solute concentration $C$ by the van't Hoff law $(\Pi=C R T$, where $R$ is the gas constant and $T$ is the absolute temperature) for an ideal solution (with very low solute concentration).

When a vesicle, which is a body of fluid surrounded by a semipermeable membrane, is placed in a solution possessing a solute concentration gradient, one pole of the vesicle experiences a higher solute concentration (and hence a higher osmotic pressure) than the opposite pole. The osmotic driving force causes solvent to cross the vesicle's membrane from inside to outside at the high concentration pole, and from outside to inside at the low concentration pole. The vesicle thus functions as a microengine, sucking fluid into it on one side and ejecting fluid on the other, thereby advancing toward regions of low concentration. This vesicle movement is termed osmophoresis (Gordon, 1981; Anderson, 1983, 1986), which provides a mechanism for the motion of biological cells in response to chemical gradients, a phenomenon known as 'chemotaxis' (Devreotes and Zigmond, 1988). An example of chemotaxis is that motile bacteria such as Escherichia coli are repelled by metabolic intermediates such as acetate (Barton and Ford, 1997). Applications of osmophoretic motion might also be found in the targeting of encapsulated drugs and other agents toward a microscopic region.

Anderson $(1983,1984)$ analyzed the osmophoretic motion of a spherical or ellipsoidal vesicle with a thin, rigid membrane through a constant solute gradient in considerable detail. He calculated the drift velocity of a spherical vesicle of radius $a$ placed in an unbounded fluid with a linear solute concentration distribution $C_{\infty}(\mathbf{x})$ far removed from the vesicle for a quite general case. In most physically realistic systems, the velocity $\mathbf{U}^{(0)}$ of a semipermeable vesicle is related to the uniform concentration gradient $\nabla C_{\infty}$ by the following expression:

$$
\mathbf{U}^{(0)}=-\frac{1}{2} a L_{\mathrm{p}} R T\left(1+\bar{\kappa}+\frac{1}{2} \kappa\right)^{-1} \nabla C_{\infty}
$$

with dimensionless parameters

$$
\begin{aligned}
& \kappa=\frac{a L_{\mathrm{p}} R T C_{\infty}\left(\mathbf{x}_{0}\right)}{D} \\
& \bar{\kappa}=\frac{a L_{\mathrm{p}} R T \bar{C}}{\bar{D}}
\end{aligned}
$$

Here, $L_{\mathrm{p}}$ is the hydraulic coefficient which is a constant for a given membrane and solvent, $\bar{D}$ 
and $D$ are the solute diffusion coefficients inside and outside the vesicle, respectively, $\bar{C}$ is the average internal concentration of solute, and $\mathbf{x}_{0}$ denotes the position of the vesicle center. The van't Hoff law was used in the derivation of Eq. (1); if it is not valid, then $R T$ must be replaced by $\partial \Pi / \partial C$, evaluated at $C_{\infty}\left(\mathbf{x}_{0}\right)$ in Eqs. (1) and (2a) and at $\bar{C}$ in Eq. (2b). Typical values in aqueous solutions for the parameters in Eq. (1) are $L_{\mathrm{p}}=10^{-9} \mathrm{~m}^{2} \mathrm{~s} / \mathrm{kg},|\nabla C \infty|=10^{5}$ $\mathrm{mol} / \mathrm{m}^{4}$ and $\kappa($ or $\bar{\kappa})=2.5$. Eq. (1) shows that the vesicle always moves toward regions of lower $C_{\infty}$, no matter what the relative values of $C_{\infty}$ and $\bar{C}$ are; that is, there exists no equilibrium position as long as $\nabla C_{\infty}$ is nonzero. Loading the inside of the vesicle with solute has a retarding effect on its velocity.

In practical applications of osmophoresis, collections of vesicles are usually encountered, and effects of vesicle interactions will be important. For example, aggregates of $E$. coli can result from a purely chemotactic response in the system containing succinate gradients (Brenner et al., 1998). Using a method of reflections, Anderson (1986) obtained analytically the migration velocity of two arbitrarily oriented identical spherical vesicles undergoing osmophoresis for the special case of $\kappa=\bar{\kappa}=0$. He also used this approximate solution for two-vesicle interactions to evaluate the mean osmophoretic velocity in a bounded suspension of identical vesicles to the leading order in the volume fraction of vesicles. On the other hand, the osmophoretic motion of two arbitrarily oriented spherical vesicles in response to a constant solute concentration gradient was examined by Keh and Yang (1992) through an exact representation in spherical bipolar coordinates. Numerical results of correction to Eq. (1) for each particle were presented for various values of the size ratio, relative separation, and parameters $\kappa$ and $\bar{\kappa}$. This numerical solution for two-vesicle interactions was also used to obtain the effect of the volume fraction of vesicles on the mean osmophoretic velocity in a bounded suspension.

In the present work, the osmophoresis of two spherical vesicles in a constant solute concentration gradient oriented arbitrarily relative to the line of vesicle centers is analyzed by using the method of reflections. The vesicles may be formed from different semipermeable membranes, contain arbitrary solute species, and have unequal radii. In Section 2 we consider the local solute concentration and fluid velocity fields produced by a single spherical vesicle placed in a prescribed concentration field whose gradient is not necessarily constant over length scales comparable to the vesicle radius. We prove that Eq. (1) also applies to a single spherical vesicle in a non-uniform solute gradient, provided that $\nabla C_{\infty}$ is evaluated at the position of the vesicle center. These results are then used in Section 3 to alternately evaluate the effects of one spherical vesicle on the other in a constant concentration gradient. The translational and angular velocities of the osmophoretic vesicles are determined in this manner with an error of $O\left(r_{12}^{-8}\right)$, where $r_{12}$ is the center-to-center distance between the vesicles, and the results are given in Eqs. (35a) and (35b). In Section 4 our method-of-reflection results are compared with the numerical calculations of Keh and Yang (1992) for the special case of two identical vesicles. Typical effects of the particle sizes and physical properties on the two-vesicle interactions in osmophoresis are discussed. Finally, in Section 5, the results of two-vesicle interactions derived in Section 3 are applied to the theory of particle concentration effects on transport properties in dilute dispersions to obtain the effect of the volume fraction of vesicles of each type on the mean osmophoretic velocities in a bounded suspension, and the general result is given in Eqs. (44) and (45). 


\section{A single vesicle in an arbitrary concentration field}

For the purpose of obtaining the interactions between two spherical vesicles undergoing osmophoresis by the method of reflections, it is necessary to obtain the solution for the migration of a single vesicle of radius $a$ in an unbounded fluid solution containing a solute species with an arbitrary concentration field $C_{A}(\mathbf{x})$. The instantaneous center of the vesicle is positioned at $\mathbf{x}_{0}$, and the relative position vector is defined as $\mathbf{r}=\mathbf{x}-\mathbf{x}_{0}\left(=r \mathbf{e}_{r}\right.$, where $\mathbf{e}_{r}$ is the unit vector in the direction of $\mathbf{r}$ ). Although $\mathbf{x}_{0}$ changes with time, the problem can be dealt with as a quasisteady state if both the Peclet and Reynolds numbers are vanishingly small. It is assumed that $a\left|\nabla C_{A}\right| / C_{A}\left(\mathbf{x}_{0}\right)<<1$.

Because the boundary conditions of the fluid velocity field are coupled with the solute concentrations at the inner and outer membrane surfaces of the vesicle, it is necessary to determine the concentration distribution first. The conservation equation governing the concentration distribution $C(\mathbf{x})$ for the external fluid of constant solute diffusion coefficient $D$ is Laplace's equation

$$
\nabla^{2} C=0
$$

For the concentration distribution $\hat{C}(\mathbf{x})$ inside the vesicle, one has

$$
\nabla^{2} \hat{C}=0
$$

It is obvious that $\nabla^{2} C_{A}=0$.

Since the radius of the vesicle is much greater than the thickness of its membrane, $r=a$ can represent both the inner and outer membrane surfaces of the vesicle. The boundary conditions require that no solute be transferred across the semipermeable membrane of the vesicle and that the concentration field far removed from the vesicle approach the prescribed values. Thus (Anderson, 1983; Keh and Yang, 1992),

$$
\begin{aligned}
& r=a: \frac{\partial \hat{C}}{\partial r}=\frac{\bar{\kappa}}{a}\left[C-C_{A}\left(\mathbf{x}_{0}\right)-(\hat{C}-\bar{C})\right] \\
& \frac{\partial C}{\partial r}=\frac{\kappa}{a}\left[C-C_{A}\left(\mathbf{x}_{0}\right)-(\hat{C}-\bar{C})\right] \\
& r \rightarrow \infty: \quad C \rightarrow C_{A}
\end{aligned}
$$

where the definition of the parameters $\kappa$ and $\bar{\kappa}$ is given by Eqs. (2a) and (2b).

A general solution to Eqs. (3a) and (3b) that satisfies Eqs. (4a), (4b) and (5) is

$$
C=C_{A}+\sum_{m=1}^{\infty}\left(\frac{a}{r}\right)^{m+1} \mathbf{S}_{m}[\cdot] \lambda_{m}
$$




$$
\hat{C}=C_{A}-C_{A}\left(\mathbf{x}_{0}\right)+\bar{C}+\sum_{m=1}^{\infty}\left(\frac{r}{a}\right)^{m} \mathbf{S}_{m}[\cdot] \hat{\lambda}_{m}
$$

Here, the $m$ th order polyadic $\mathbf{S}_{m}$ is a surface harmonic, defined by

$$
\mathbf{S}_{m}=r^{m+1} \nabla^{m}\left(r^{-1}\right)
$$

The first few ones are

$$
\begin{aligned}
& \mathbf{S}_{0}=1 \\
& \mathbf{S}_{1}=-\frac{\mathbf{r}}{r} \\
& \mathbf{S}_{2}=3 \frac{\mathbf{r r}}{r^{2}}-\mathbf{I}
\end{aligned}
$$

where $\mathbf{I}$ is the unit dyadic. $\lambda_{m}$ and $\hat{\lambda}_{m}$ are $m$ th order polyadic constants, and the symbol [ $\cdot$ ] represents $m$ scalar products using the inner nesting convention.

Substitution of Eqs. (6a) and (6b) into Eqs. (4a) and (4b) leads to

$$
\begin{aligned}
& \lambda_{1}=-G a\left(\nabla C_{A}\right)_{0} \\
& \lambda_{2}=\frac{1}{3} H a^{2}\left(\nabla \nabla C_{A}\right)_{0} \\
& \hat{\lambda}_{1}=-\hat{G} a\left(\nabla C_{A}\right)_{0} \\
& \hat{\lambda}_{2}=\frac{1}{3} \hat{H} a^{2}\left(\nabla \nabla C_{A}\right)_{0}
\end{aligned}
$$

where

$$
\begin{aligned}
& G=\frac{1+\bar{\kappa}-\kappa}{2+2 \bar{\kappa}+\kappa} \\
& H=\frac{2+\bar{\kappa}-\kappa}{6+3 \bar{\kappa}+2 \kappa} \\
& \hat{G}=\frac{-2+\bar{\kappa}-\kappa}{2+2 \bar{\kappa}+\kappa} \\
& \hat{H}=\frac{-3+\bar{\kappa}-\kappa}{6+3 \bar{\kappa}+2 \kappa}
\end{aligned}
$$

and the subscript 0 to variables inside parentheses denotes evaluation at $\mathbf{x}=\mathbf{x}_{0}$. Substituting 
Eqs. (9a)-(9d) into Eqs. (6a) and (6b) and realizing that $C_{A}$ satisfies Laplace's equation, we obtain

$$
\begin{aligned}
& C=C_{A}+G\left(\frac{a}{r}\right)^{3} \mathbf{r} \cdot\left(\nabla C_{A}\right)_{0}+H\left(\frac{a}{r}\right)^{5} \mathbf{r r}:\left(\nabla \nabla C_{A}\right)_{0}+O\left(\nabla \nabla \nabla C_{A}\right)_{0} \\
& \hat{C}=C_{A}-C_{A}\left(\mathbf{x}_{0}\right)+\bar{C}+\hat{G} \mathbf{r} \cdot\left(\nabla C_{A}\right)_{0}+\hat{H} \mathbf{r r}:\left(\nabla \nabla C_{A}\right)_{0}+O\left(\nabla \nabla \nabla C_{A}\right)_{0}
\end{aligned}
$$

Knowing the solute concentration distributions inside and outside the vesicle, we can now take up the solution of the fluid velocity field. Due to the low Reynolds number encountered in the osmophoresis, the fluid velocity is governed by the Stokes equations

$$
\begin{aligned}
& \eta \nabla^{2} \mathbf{v}-\nabla p=\mathbf{0} \\
& \nabla \cdot \mathbf{v}=0
\end{aligned}
$$

where $\mathbf{v}(\mathbf{x})$ is the fluid velocity, $p(\mathbf{x})$ is the dynamic pressure, and $\eta$ is the fluid viscosity.

The boundary condition for the fluid velocity at the surface of the vesicle is (Anderson, 1983; Keh and Yang, 1992)

$$
r=a: \quad \mathbf{v}=\mathbf{v}_{S}=\mathbf{U}+a \mathbf{\Omega} \times \mathbf{e}_{r}+L_{\mathrm{P}} R T\left[C-C_{A}\left(\mathbf{x}_{0}\right)-(\hat{C}-\bar{C})\right] \mathbf{e}_{r}
$$

where $\mathbf{U}$ and $\boldsymbol{\Omega}$ are the instantaneous translational and angular velocities, respectively, of the vesicle to be determined. The solute concentration distributions $C$ and $\hat{C}$ in Eq. (13) have been obtained in Eqs. (11a) and (11b). Since the fluid is at rest far away from the vesicle, one has

$$
r \rightarrow \infty: \quad \mathbf{v} \rightarrow \mathbf{0}
$$

A solution to Eqs. (12)-(14) for the velocity field can be constructed from Lamb's general solution as outlined by Brenner (1964). The fluid velocity is completely specified when the polyadic coefficients $\boldsymbol{\alpha}_{m}, \boldsymbol{\beta}_{m}$ and $\boldsymbol{\gamma}_{m}$ in the following formulas are calculated using the value of the velocity field on the surface of the vesicle $\left(\mathbf{v}_{S}\right)$ :

$$
\begin{aligned}
& \mathbf{e}_{r} \cdot \mathbf{v}_{S}=\sum_{m=1}^{\infty} \boldsymbol{\alpha}_{m}[\cdot] \mathbf{S}_{m} \\
& -a \nabla \cdot \mathbf{v}_{S}=\sum_{m=1}^{\infty} \boldsymbol{\beta}_{m}[\cdot] \mathbf{S}_{m} \\
& a \mathbf{e}_{r} \cdot\left(\nabla \times \mathbf{v}_{S}\right)=\sum_{m=1}^{\infty} \boldsymbol{\gamma}_{m}[\cdot] \mathbf{S}_{m}
\end{aligned}
$$

The force and torque exerted by the surrounding fluid on the surface $r=a$ are given by

$$
\mathbf{F}=2 \pi \eta a\left(3 \boldsymbol{\alpha}_{1}+\boldsymbol{\beta}_{1}\right)
$$




$$
\mathbf{T}=4 \pi \eta a^{2} \boldsymbol{\gamma}_{1}
$$

Since the vesicle is freely suspended in the fluid, the velocities $\mathbf{U}$ and $\boldsymbol{\Omega}$ are obtained by setting the above expressions for $\mathbf{F}$ and $\mathbf{T}$ equal to zero.

Substitution of Eq. (13) into the left-hand side of Eq. (15c) yields

$$
\begin{aligned}
& \boldsymbol{\gamma}_{1}=-2 a \boldsymbol{\Omega} \\
& \boldsymbol{\gamma}_{m}=\mathbf{0} \text { for } m>1
\end{aligned}
$$

By setting $\mathbf{T}=\mathbf{0}$ and using Eqs. (16b) and (17b), one finds

$$
\mathbf{\Omega}=\mathbf{0}
$$

as the results for a prescribed solute concentration field $C_{A}$ as long as $\nabla^{2} C_{A}=0$.

The translational motion is described by the coefficient $\boldsymbol{\alpha}_{m}$ and $\boldsymbol{\beta}_{m}$. It can be obtained from Eqs. (11a), (11b), (13), and (15a) that

$$
\begin{aligned}
& \boldsymbol{\alpha}_{1}=-\mathbf{U}-a L_{\mathrm{p}} R T(G-\hat{G})\left(\nabla C_{A}\right)_{0} \\
& \boldsymbol{\alpha}_{2}=\frac{1}{3} a^{2} L_{\mathrm{p}} R T(H-\hat{H})\left(\nabla \nabla C_{A}\right)_{0} \\
& \boldsymbol{\alpha}_{m}=\mathbf{0} \text { for } m>2
\end{aligned}
$$

Finally, substituting Eq. (13) together with Eqs. (11a) and (11b) into Eq. (15b) and neglecting the terms of $O\left(\nabla \nabla \nabla C_{A}\right)_{0}$ (only $\boldsymbol{\beta}_{1}$ and $\boldsymbol{\beta}_{2}$ are needed in the following calculations), one has

$$
\begin{aligned}
& \boldsymbol{\beta}_{1}=2 a L_{\mathrm{p}} R T(G-\hat{G})\left(\nabla C_{A}\right)_{0} \\
& \boldsymbol{\beta}_{2}=-\frac{2}{3} a^{2} L_{\mathrm{p}} R T(H-\hat{H})\left(\nabla \nabla C_{A}\right)_{0}
\end{aligned}
$$

The force-free characteristic of this problem is used with Eqs. (16a), (19a), and (20a), and the translational velocity is found to be

$$
\mathbf{U}=A\left(\nabla C_{A}\right)_{0}
$$

where

$$
A=-\frac{1}{3} a L_{\mathrm{p}} R T(G-\hat{G})=-\frac{a L_{\mathrm{p}} R T}{2+2 \bar{\kappa}+\kappa}
$$

Eqs. (21) and (22) show that the osmophoretic velocity of the vesicle is proportional to the prescribed solute concentration gradient evaluated at the vesicle center and is identical to Eq. (1) in the dependence on the properties of the solution and the vesicle itself.

The corresponding velocity field external to the osmophoretic vesicle is determined by 


$$
\mathbf{v}=\nabla \phi_{-2}+\nabla \phi_{-3}+\frac{1}{2 \eta} \mathbf{r} p_{-3}+O\left(\nabla \nabla \nabla C_{A}\right)_{0}
$$

where the solid spherical harmonic functions $\phi_{-(m+1)}$ and $p_{-(m+1)}$ are (Happel and Brenner, 1983),

$$
\begin{aligned}
& \phi_{-2}=\frac{a^{3}}{4} r^{-2}\left(\boldsymbol{\alpha}_{1}+\boldsymbol{\beta}_{1}\right) \cdot\left(-\mathbf{e}_{r}\right) \\
& \phi_{-3}=\frac{a^{4}}{6} r^{-3}\left(2 \boldsymbol{\alpha}_{2}+\boldsymbol{\beta}_{2}\right):\left(3 \mathbf{e}_{r} \mathbf{e}_{r}-\mathbf{I}\right) \\
& p_{-3}=\eta a^{2} r^{-3}\left(4 \boldsymbol{\alpha}_{2}+\boldsymbol{\beta}_{2}\right):\left(3 \mathbf{e}_{r} \mathbf{e}_{r}-\mathbf{I}\right), \text { etc. }
\end{aligned}
$$

After evaluating $\phi_{-2}, \phi_{-3}$, and $p_{-3}$ from $\boldsymbol{\alpha}_{1}, \boldsymbol{\beta}_{1}, \boldsymbol{\alpha}_{2}$, and $\boldsymbol{\beta}_{2}$, we obtain the external velocity field as

$$
\mathbf{v}=-A\left(\frac{a}{r}\right)^{3}\left(3 \frac{\mathbf{r r}}{r^{2}}-\mathbf{I}\right) \cdot\left(\nabla C_{A}\right)_{0}+B\left(\frac{a}{r}\right)^{3} \frac{\mathbf{r r r}}{r^{2}}:\left(\nabla \nabla C_{A}\right)_{0}+O\left(\nabla \nabla \nabla C_{A}\right)_{0}
$$

where

$$
B=a L_{\mathrm{p}} R T(H-\hat{H})=\frac{5 a L_{\mathrm{p}} R T}{6+3 \bar{\kappa}+2 \kappa}
$$

For the motion of a freely-suspended spherical vesicle under arbitrary imposed solute concentration gradient $\nabla C_{A}$ and velocity field $\mathbf{v}_{A}$ in an unbounded fluid, the translational and angular velocities of the vesicle can be obtained by linearly combining the Faxen laws for a force-free and torque-free sphere with no-slip surface (Happel and Brenner, 1983), Eqs. (18) and (21),

$$
\begin{aligned}
& \mathbf{U}=A\left(\nabla C_{A}\right)_{0}+\left(\mathbf{v}_{A}\right)_{0}+\frac{1}{6} a^{2}\left(\nabla^{2} \mathbf{v}_{A}\right)_{0} \\
& \mathbf{\Omega}=\frac{1}{2}\left(\nabla \times \mathbf{v}_{A}\right)_{0}
\end{aligned}
$$

Note that the vesicle behaves as an impermeable particle in the applied velocity field $\mathbf{v}_{A}$ because the normal fluid velocity through the vesicle surface does not exist due to the fact that both the normal component of the velocity gradient and the dynamic pressure are continuous across the zero-thickness membrane and the viscosity is the same for both the internal and external fluids.

\section{Solution for interactions between two osmophoretic vesicles}

In this section we consider the quasisteady osmophoresis of two spherical vesicles of radii $a_{1}$ 
and $a_{2}$. They are oriented at an arbitrary angle to the prescribed solute concentration gradient $\mathbf{E}_{\infty}\left(=\nabla C_{\infty}\right.$, where $C_{\infty}(\mathbf{x})$ is the undisturbed concentration field of the surrounding solution). The vesicles, which may be formed from different semipermeable membranes and contain arbitrary solutes, are supposed to be sufficiently close to interact with each other, but sufficiently distant from boundary walls for the ambient fluid to be regarded as unbounded. Let $\mathbf{e}$ be the unit vector pointing from the center of vesicle 1 to the center of vesicle 2 and $r_{12}$ be the center-to-center distance between the vesicles. $\mathbf{E}_{\infty}$ is assumed to be constant over distances comparable to $r_{12}$ and the fluid at infinity is at rest. The objective is to determine the correction to Eq. (1) for the motion of each vesicle due to the presence of the other.

In the situation $\left(a_{1}+a_{2}\right) / r_{12}<<1$, a method of reflections (Anderson, 1985; Chen and Keh, 1988 ) is used to solve the two-sphere problem. Because of the linear characteristic of the governing equations (3a), (3b), (12a) and (12b) as well as the boundary conditions, Eqs. (4a), (4b), (5), (13) and (14), the solution of the solute concentration and fluid velocity fields external to the vesicles for Eqs. (3a) and (12a) and (12b) can be decomposed into a sum of fields, which depend on increasing powers of $r_{12}^{-1}$,

$$
\begin{aligned}
& C=C_{1}^{(1)}+C_{2}^{(2)}+C_{1}^{(3)}+C_{2}^{(4)}+\cdots \\
& \mathbf{v}=\mathbf{v}_{1}^{(1)}+\mathbf{v}_{2}^{(2)}+\mathbf{v}_{1}^{(3)}+\mathbf{v}_{2}^{(4)}+\cdots
\end{aligned}
$$

where subscripts 1 and 2 represent the reflections from vesicle 1 and vesicle 2, respectively, and the superscript $(i)$ denotes the $i$ th reflection from either vesicle surface. Hence, the vesicles' translational and angular velocities can also be expressed in the form of a series,

$$
\begin{aligned}
& \mathbf{U}_{1}=\mathbf{U}_{1}^{(0)}+\mathbf{U}_{1}^{(2)}+\mathbf{U}_{1}^{(4)}+\cdots \\
& \mathbf{\Omega}_{1}=\mathbf{\Omega}_{1}^{(0)}+\mathbf{\Omega}_{1}^{(2)}+\mathbf{\Omega}_{1}^{(4)}+\cdots \\
& \mathbf{U}_{2}=\mathbf{U}_{2}^{(1)}+\mathbf{U}_{2}^{(3)}+\mathbf{U}_{2}^{(5)}+\cdots \\
& \mathbf{\Omega}_{2}=\mathbf{\Omega}_{2}^{(1)}+\mathbf{\Omega}_{2}^{(3)}+\mathbf{\Omega}_{2}^{(5)} \cdots
\end{aligned}
$$

where $\mathbf{U}_{1}^{(i)}$ and $\boldsymbol{\Omega}_{1}^{(i)}$ are related to $C_{2}^{(i)}$ and $\mathbf{v}_{2}^{(i)}$ by Eqs. (27a) and (27b) for $i=2,4,6, \ldots$, while $\mathbf{U}_{2}^{(i)}$ and $\boldsymbol{\Omega}_{2}^{(i)}$ are related to $C_{1}^{(i)}$ and $\mathbf{v}_{1}^{(i)}$ for $i=1,3,5, \ldots$ Obviously, the unperturbed linear concentration field gives

$$
\begin{aligned}
& \mathbf{U}_{1}^{(0)}=A_{1} \mathbf{E}_{\infty} \\
& \mathbf{\Omega}_{1}^{(0)}=\mathbf{0}
\end{aligned}
$$

Hereinafter, we use $A_{j}, B_{j}$ and $G_{j}$ to represent the values of $A, B$ and $G$, respectively, defined by Eqs. (22), (26), and (10a) for the vesicle $j(j=1$ or 2$)$.

The initial concentration gradient $\nabla C_{1}^{(1)}$ and velocity field $\mathbf{v}_{1}^{(1)}$, which correspond to the 
osmophoresis of vesicle 1 isolated in an unbounded fluid under the prescribed field $\mathbf{E}_{\infty}$, are easily obtained from Eqs. (11a) and (25) for $r_{1}>a_{1}$ as

$$
\begin{aligned}
& \nabla C_{1}^{(1)}=\mathbf{E}_{\infty}-G_{1}\left(\frac{a_{1}}{r_{1}}\right)^{3}\left(3 \frac{\mathbf{r}_{1} \mathbf{r}_{1}}{r_{1}^{2}}-\mathbf{I}\right) \cdot \mathbf{E}_{\infty} \\
& \mathbf{v}_{1}^{(1)}=-A_{1}\left(\frac{a_{1}}{r_{1}}\right)^{3}\left(3 \frac{\mathbf{r}_{1} \mathbf{r}_{1}}{r_{1}^{2}}-\mathbf{I}\right) \cdot \mathbf{E}_{\infty}
\end{aligned}
$$

where $\mathbf{r}_{1}$ is the position vector relative to the center of vesicle 1 and $r_{1}=\left|\mathbf{r}_{1}\right|$. Note that $\mathbf{v}_{1}^{(1)}$ is irrotational and satisfies Laplace's equation. The contributions of $C_{1}^{(1)}$ and $\mathbf{v}_{1}^{(1)}$ to the velocity of vesicle 2 (with the center at position $\mathbf{r}_{1}=r_{12} \mathbf{e}$ ) are determined from Eqs. (27a) and (27b) taking $C_{A}=C_{1}^{(1)}$ and $\mathbf{v}_{A}=\mathbf{v}_{1}^{(1)}$. Thus,

$$
\begin{aligned}
& \mathbf{U}_{2}^{(1)}=A_{2} \mathbf{E}_{\infty}-\left(A_{1}+A_{2} G_{1}\right)\left(\frac{a_{1}}{r_{12}}\right)^{3}(3 \mathbf{e e}-\mathbf{I}) \cdot \mathbf{E}_{\infty} \\
& \mathbf{\Omega}_{2}^{(1)}=\mathbf{0}
\end{aligned}
$$

Eq. (32a) indicates that the effect of vesicle interaction in osmophoresis is $O\left(r_{12}^{-3}\right)$.

The first reflected concentration gradient field from vesicle 2 can be derived from using Eqs. (11a) and (31a), while the first reflected velocity field from vesicle 2 can be determined from Eqs. (25), (31a) and (31b), and the analysis of Chen and Keh (1988). The results are

$$
\begin{aligned}
& \nabla C_{2}^{(2)}=-G_{2}\left(\frac{a_{2}}{r_{2}}\right)^{3}\left(3 \frac{\mathbf{r}_{2} \mathbf{r}_{2}}{r_{2}^{2}}-\mathbf{I}\right) \cdot\left(\nabla C_{1}^{(1)}\right)_{\mathbf{r}_{1}=\mathbf{r}_{12} \mathbf{e}}+O\left(r_{2}^{-4} \nabla \nabla C_{1}^{(1)}+r_{2}^{-3} \nabla \nabla \nabla C_{1}^{(1)}\right) \\
& \mathbf{v}_{2}^{(2)}=-A_{2}\left(\frac{a_{2}}{r_{2}}\right)^{3}\left(3 \frac{\mathbf{r}_{2} \mathbf{r}_{2}}{r_{2}^{2}}-\mathbf{I}\right) \cdot\left(\nabla C_{1}^{(1)}\right)_{\mathbf{r}_{1}=\mathbf{r}_{12} \mathbf{e}}+B_{2}\left(\frac{a_{2}}{r_{2}}\right)^{3} \frac{\mathbf{r}_{2} \mathbf{r}_{2} \mathbf{r}_{2}}{r_{2}^{2}}: \quad\left(\nabla \nabla C_{1}^{(1)}\right)_{\mathbf{r}_{1}=\mathbf{r}_{12} \mathbf{e}} \\
& \quad-\frac{5}{2}\left(\frac{a_{2}}{r_{2}}\right)^{3} \frac{\mathbf{r}_{2} \mathbf{r}_{2} \mathbf{r}_{2}}{r_{2}^{2}}:\left(\nabla \mathbf{v}_{1}^{(1)}\right)_{\mathbf{r}_{1}=\mathbf{r}_{12} \mathbf{e}}+O\left[r_{2}^{-4}\left(\nabla \nabla C_{1}^{(1)}+\nabla \mathbf{v}_{1}^{(1)}\right)+r_{2}^{-3}\left(\nabla \nabla \nabla C_{1}^{(1)}+\nabla \nabla \mathbf{v}_{1}^{(1)}\right)\right]
\end{aligned}
$$

Substituting Eqs. (33a) and (33b) into Eqs. (27a) and (27b) with $C_{A}=C_{2}^{(2)}$ and $\mathbf{v}_{A}=\mathbf{v}_{2}^{(2)}$, one obtains the contribution to the velocity of vesicle 1 due to the reflected fields from vesicle 2 ,

$$
\begin{aligned}
\mathbf{U}_{1}^{(2)}= & -\left(A_{2}+A_{1} G_{2}\right)\left(\frac{a_{2}}{r_{12}}\right)^{3}(3 \mathbf{e e}-\mathbf{I}) \cdot \mathbf{E}_{\infty}+\left[\left(A_{1} G_{1} G_{2}+A_{2} G_{1}\right)(3 \mathbf{e e}+\mathbf{I})\right. \\
& \left.+3\left(5 A_{1}-2 B_{2} G_{1}\right) \mathbf{e e}\right] \frac{a_{1}^{3} a_{2}^{3}}{r_{12}^{6}} \cdot \mathbf{E}_{\infty}+O\left(r_{12}^{-8}\right)
\end{aligned}
$$




$$
\mathbf{\Omega}_{1}^{(2)}=3\left(5 A_{1}-2 B_{2} G_{1}\right) \frac{a_{1}^{3} a_{2}^{3}}{r_{12}^{7}} \mathbf{e} \times \mathbf{E}_{\infty}+O\left(r_{12}^{-9}\right)
$$

Obviously, $\mathbf{U}_{1}^{(4)}$ and $\boldsymbol{\Omega}_{1}^{(4)}$ will be of the orders $O\left(r_{12}^{-9}\right)$ and $O\left(r_{12}^{-10}\right)$, respectively. With the addition of Eqs. (30) and (34), the translational and angular velocities of vesicle 1 can be expressed as

$$
\begin{aligned}
\mathbf{U}_{1}= & A_{1} \mathbf{E}_{\infty}-\left(A_{2}+A_{1} G_{2}\right)\left(\frac{a_{2}}{r_{12}}\right)^{3}(3 \mathbf{e e}-\mathbf{I}) \cdot \mathbf{E}_{\infty}+\left[\left(A_{1} G_{1} G_{2}+A_{2} G_{1}\right)(3 \mathbf{e e}+\mathbf{I})\right. \\
& \left.+3\left(5 A_{1}-2 B_{2} G_{1}\right) \mathbf{e e}\right] \frac{a_{1}^{3} a_{2}^{3}}{r_{12}^{6}} \cdot \mathbf{E}_{\infty}+O\left(r_{12}^{-8}\right) \\
\mathbf{\Omega}_{1}= & 3\left(5 A_{1}-2 B_{2} G_{1}\right) \frac{a_{1}^{3} a_{2}^{3}}{r_{12}^{7}} \mathbf{e} \times \mathbf{E}_{\infty}+O\left(r_{12}^{-9}\right)
\end{aligned}
$$

$\mathbf{U}_{2}$ and $\boldsymbol{\Omega}_{2}$, the velocities of vesicle 2 , can be obtained from the above formulas by interchanging the subscripts 1 and 2 in all variables and replacing e by $-\mathbf{e}$. As expected, both vesicles will move with the velocity that would exist in the absence of the other (without rotation) for any orientation of the vesicles as $r_{12} \rightarrow \infty$.

\section{Discussion on interactions between two vesicles}

The interaction between two vesicles in a solute concentration gradient field, given by Eqs. (35a) and (35b), results from two phenomena: each vesicle disturbs the local concentration field experienced by the other, and the movement of each vesicle generates a fluid velocity field that convects and rotates the other. The leading term of the interaction for vesicle translation is $O\left(r_{12}^{-3}\right)$, because both the concentration gradient and velocity disturbances in the surrounding fluid produced by an isolated osmophoretic vesicle decay like $r^{-3}$, as shown in Eqs. (11a) and (25). For osmophoretic vesicles that allow free rotation, the leading term of the angular velocity is of $O\left(r_{12}^{-7}\right)$. Thus, the interaction between vesicles undergoing osmophoresis is much weaker than that between sedimenting particles, since the leading terms of particle interaction for the translational and angular velocities of two spheres driven by body forces are of $O\left(r_{12}^{-1}\right)$ and $O\left(r_{12}^{-2}\right)$, respectively (Happel and Brenner, 1983).

The exact (numerical) solution for the problem of osmophoresis of two arbitrary spherical vesicles was obtained by using bipolar coordinates (Keh and Yang, 1992). Tables 1 and 2 give the comparisons in osmophoretic velocities of the two vesicles with the line of their centers parallel and perpendicular, respectively, to the applied solute concentration gradient $\mathbf{E}_{\infty}$. For simplicity, only the case of two identical vesicles $\left(a_{1}=a_{2}=a, L_{\mathrm{p} 1}=L_{\mathrm{p} 2}=L_{\mathrm{p}}, \kappa_{1}=\kappa_{2}=\kappa\right.$, $\left.\bar{\kappa}_{1}=\bar{\kappa}_{2}=\bar{\kappa}, U_{1}^{(0)}=U_{2}^{(0)}=U^{(0)}=\left|\mathbf{U}^{(0)}\right|\right)$ is presented. In this specific case, the vesicles will migrate at the same velocity $\left(U_{1}=U_{2}=U\right)$ and rotate with equal but opposite angular velocities $\left(\Omega_{1}=-\Omega_{2}=\Omega\right)$. It is found in Tables 1 and 2 that the predictions of the normalized velocities $U / U^{(0)}$ and $a \Omega / U^{(0)}$ from the asymptotic approximation given by Eqs. (35a) and (35b) for 


\section{Table 1}

Normalized osmophoretic velocities $U / U^{(0)}$ of two identical vesicles with the line through their centers aligned with the prescribed solute concentration gradient

\begin{tabular}{|c|c|c|c|c|}
\hline \multirow[b]{2}{*}{$\kappa$} & \multirow[b]{2}{*}{$\bar{\kappa}$} & \multirow[b]{2}{*}{$2 a / r_{12}$} & \multicolumn{2}{|l|}{$U / U^{(0)}$} \\
\hline & & & Asymptotic solution & Exact solution \\
\hline \multirow[t]{5}{*}{0} & \multirow[t]{5}{*}{0} & 0.2 & 0.99702 & 0.99702 \\
\hline & & 0.4 & 0.97747 & 0.97747 \\
\hline & & 0.6 & 0.93577 & 0.93472 \\
\hline & & 0.8 & 0.90221 & 0.88241 \\
\hline & & 0.9 & 0.91761 & 0.85879 \\
\hline \multirow[t]{5}{*}{0} & \multirow[t]{5}{*}{5} & 0.2 & 0.99702 & 0.99703 \\
\hline & & 0.4 & 0.97770 & 0.97771 \\
\hline & & 0.6 & 0.93837 & 0.93737 \\
\hline & & 0.8 & 0.91684 & 0.89429 \\
\hline & & 0.9 & 0.94727 & 0.87805 \\
\hline \multirow[t]{5}{*}{5} & \multirow[t]{5}{*}{0} & 0.2 & 0.99915 & 0.99915 \\
\hline & & 0.4 & 0.99356 & 0.99357 \\
\hline & & 0.6 & 0.98161 & 0.98145 \\
\hline & & 0.8 & 0.97185 & 0.96743 \\
\hline & & 0.9 & 0.97604 & 0.96210 \\
\hline \multirow[t]{5}{*}{5} & \multirow[t]{5}{*}{5} & 0.2 & 0.99790 & 0.99790 \\
\hline & & 0.4 & 0.98410 & 0.98410 \\
\hline & & 0.6 & 0.95465 & 0.95403 \\
\hline & & 0.8 & 0.93090 & 0.91784 \\
\hline & & 0.9 & 0.94169 & 0.90198 \\
\hline
\end{tabular}

various values of $\kappa$ and $\bar{\kappa}$ are in good agreement with those of the exact solution. The errors in $U / U^{(0)}$ are less than $0.12 \%$ for cases $2 a / r_{12} \leqslant 0.6$ or $2.6 \%$ for cases $2 a / r_{12} \leqslant 0.8$ (although the errors in $a \Omega / U^{(0)}$ are relatively large), indicating that the higher terms such as $O\left(r_{12}^{-8}\right)$ in Eq. (35a) are not important unless the vesicles are nearly touching. Note that the interaction effect between the vesicles evaluated from Eqs. (35a) and (35b) always overestimates the value of $U /$ $U^{(0)}$ and underestimates the value of $a \Omega / U^{(0)}$. For the case of two vesicles differing in size and/ or in physical properties, Eqs. (35a) and (35b) can also be found to agree well with the exact solution.

The translational and rotational velocities for various cases of two spherical vesicles evaluated from Eqs. (35a) and (35b) are shown by solid curves in Figs. 1-3. The corresponding numerical results (with finite values of $\kappa$ and $\bar{\kappa}$ ) obtained by using bipolar coordinates (Keh and Yang, 1992) are also presented by dashed curves in these figures for comparison purposes. In each case, the velocity of a vesicle normalized by the value that prevails in the absence of the other is plotted versus the separation parameter $\left(a_{1}+a_{2}\right) / r_{12}$. It can be seen that the effect of particle interactions on the normalized osmophoretic velocities in general is increased with the increase in $\left(a_{1}+a_{2}\right) / r_{12}$.

For the situation of two identical vesicles oriented along the prescribed solute concentration gradient, the interaction effect makes each vesicle move slower than its undisturbed value, as 
Table 2

Normalized osmophoretic velocities $U / U^{(0)}$ and $a \Omega / U^{(0)}$ of two identical vesicles with the line through their centers perpendicular to the prescribed solute concentration gradient

\begin{tabular}{|c|c|c|c|c|c|c|}
\hline \multirow[b]{2}{*}{$\kappa$} & \multirow[b]{2}{*}{$\bar{\kappa}$} & \multirow[b]{2}{*}{$2 a / r_{12}$} & \multicolumn{2}{|c|}{ Asymptotic solution } & \multicolumn{2}{|c|}{ Exact solution } \\
\hline & & & $U / U^{(0)}$ & $a \Omega / U^{(0)}$ & $U / U^{(0)}$ & $a \Omega / U^{(0)}$ \\
\hline \multirow[t]{5}{*}{0} & \multirow[t]{5}{*}{0} & 0.2 & 1.00150 & $1.0 \times 10^{-6}$ & 1.00150 & $1.0 \times 10^{-6}$ \\
\hline & & 0.4 & 1.01205 & 0.00013 & 1.01204 & 0.00015 \\
\hline & & 0.6 & 1.04105 & 0.00219 & 1.04075 & 0.00332 \\
\hline & & 0.8 & 1.09907 & 0.01638 & 1.09325 & 0.04136 \\
\hline & & 0.9 & 1.14292 & 0.03737 & 1.11681 & 0.15474 \\
\hline \multirow[t]{5}{*}{0} & \multirow[t]{5}{*}{5} & 0.2 & 1.00150 & $1.2 \times 10^{-6}$ & 1.00150 & $1.5 \times 10^{-6}$ \\
\hline & & 0.4 & 1.01205 & 0.00015 & 1.01204 & 0.00018 \\
\hline & & 0.6 & 1.04105 & 0.00258 & 1.04074 & 0.00400 \\
\hline & & 0.8 & 1.09907 & 0.01931 & 1.09243 & 0.05155 \\
\hline & & 0.9 & 1.14292 & 0.04404 & 1.11024 & 0.20354 \\
\hline \multirow[t]{5}{*}{5} & \multirow[t]{5}{*}{0} & 0.2 & 1.00043 & $3.8 \times 10^{-7}$ & 1.00043 & $3.9 \times 10^{-7}$ \\
\hline & & 0.4 & 1.00341 & 0.00005 & 1.00341 & 0.00006 \\
\hline & & 0.6 & 1.01139 & 0.00082 & 1.01119 & 0.00127 \\
\hline & & 0.8 & 1.02643 & 0.00614 & 1.02302 & 0.01498 \\
\hline & & 0.9 & 1.03902 & 0.01401 & 1.02467 & 0.04977 \\
\hline \multirow[t]{5}{*}{5} & \multirow[t]{5}{*}{5} & 0.2 & 1.00106 & $8.0 \times 10^{-6}$ & 1.00106 & $8.3 \times 10^{-6}$ \\
\hline & & 0.4 & 1.00848 & 0.00010 & 1.00847 & 0.00012 \\
\hline & & 0.6 & 1.02863 & 0.00175 & 1.02830 & 0.00267 \\
\hline & & 0.8 & 1.06802 & 0.01308 & 1.06211 & 0.03254 \\
\hline & & 0.9 & 1.09700 & 0.02983 & 1.07295 & 0.11696 \\
\hline
\end{tabular}

illustrated in Figs. 1(a) and 2(a). On the other hand, the normalized migration velocity of two identical vesicles undergoing osmophoresis normal to the line of their centers is a monotonic increasing function of the separation parameter $2 a / r_{12}$, as illustrated in Figs. 1(b) and 2(b). A careful examination of the surrounding fluid recirculation pattern generated by the osmophoretic motion of a single spherical vesicle (Anderson, 1986) shows why the particle interactions cause the enhancement or retardation in the vesicle velocity. In Figs. 1(c) and 2(c), the normalized angular velocities of two identical vesicles undergoing osmophoresis normal to the line of their centers are plotted versus $2 a / r_{12}$ with $\kappa$ and $\bar{\kappa}$ as parameters. For two spheres aligned with the prescribed solute concentration gradient the flow is axisymmetric and the angular velocities of vesicles vanish. Figs. 1 and 2 indicate that the vesicle interaction effect is more significant if the value of $\kappa$ becomes smaller or the value of $\bar{\kappa}$ becomes greater, keeping the other as a constant. In the limit $\kappa \rightarrow \infty$, Eqs. (10a), (22), and (26) give $G=-1$ and $B /$ $A=-5 / 2$. Under this situation, the osmophoretic velocity of each of these two identical vesicles (which can be arbitrarily oriented) is unaffected by the presence of the other, as predicted by Eqs. (35a) and (35b).

In Fig 3, the normalized translational and rotational velocities (of vesicle 1) for the osmophoresis of two unequally sized vesicles with the same physical properties $\left(L_{\mathrm{p}}, \kappa\right.$ and $\left.\bar{\kappa}\right)$ are plotted versus $\left(a_{1}+a_{2}\right) / r_{12}$ with the ratio $a_{2} / a_{1}$ as a parameter for the case of $\kappa=\bar{\kappa}=0$. 

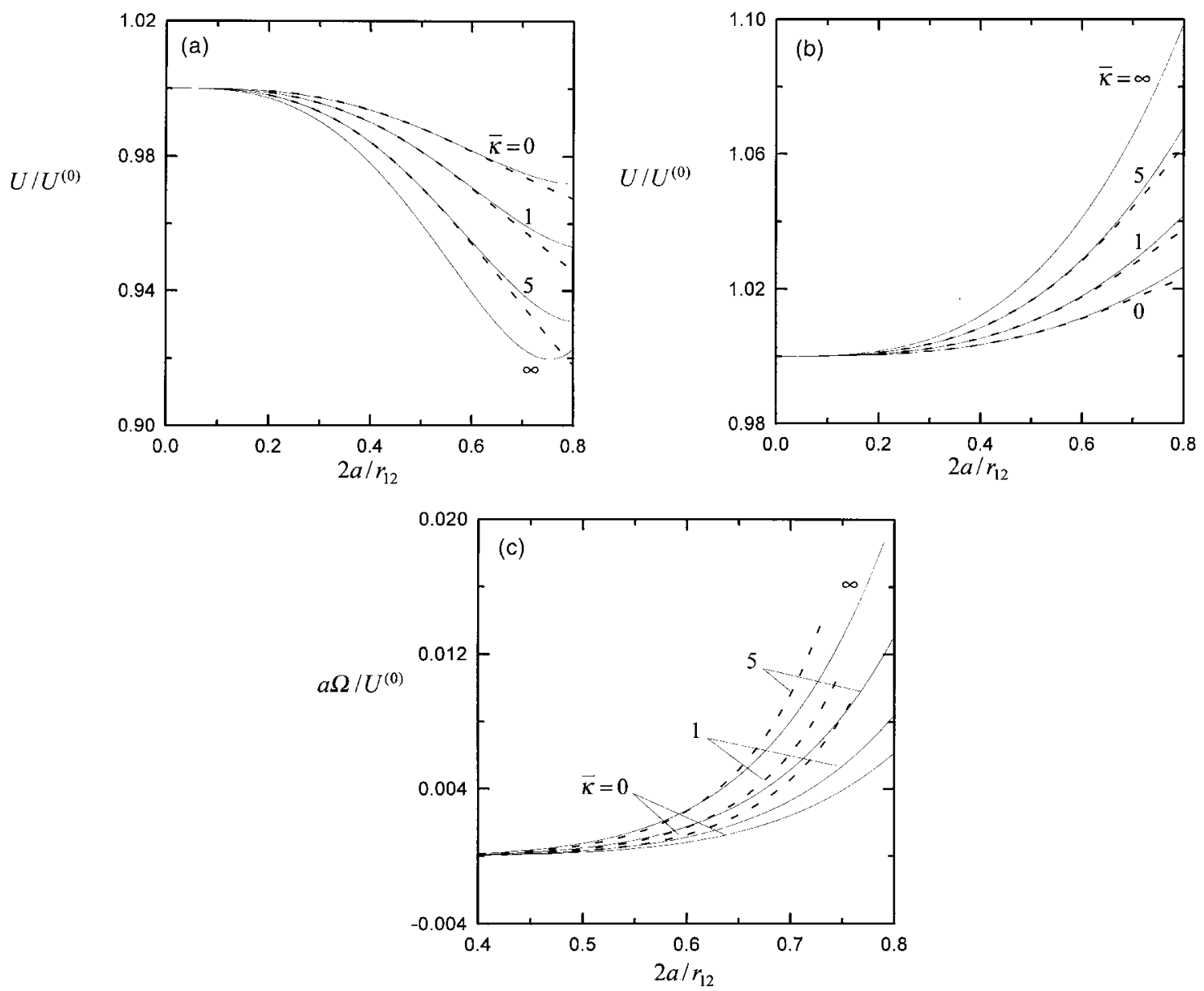

Fig. 1. Normalized translational and rotational velocities of two identical vesicles versus the separation parameter $2 a / r_{12}$ with $\kappa=5$ and $\bar{\kappa}$ as a parameter: (a) solute concentration gradient prescribed parallel to the line of vesicle centers; (b) and (c) solute concentration gradient prescribed perpendicular to the line of vesicle centers. The solid curves are computed from Eqs. (35a) and (35b), while the dashed curves are results obtained using bipolar coordinates.

Again, this figure indicates that the migration velocity of the first vesicle in general is reduced if the second vesicle is oriented along the imposed solute concentration gradient (with some exceptions when $a_{2} / a_{1}$ is small) and is enhanced if the second one is oriented perpendicular to the gradient. It can be seen that the effect of vesicle interactions on the normalized osmophoretic mobility is far greater on the smaller vesicle than on the larger one. For example, when the larger vesicle has a radius three times that of the smaller one, the migration velocity of the smaller vesicle [with $\left(a_{1}+a_{2}\right) / r_{12}=0.8$ ] can be decreased by as much as $45 \%$ (if the concentration gradient is applied along the line of vesicle centers) or increased by $75 \%$ (if the concentration gradient is prescribed normal to the line of vesicle centers), while the migration velocity of the large vesicle can hardly be influenced even when the two vesicles are nearly 

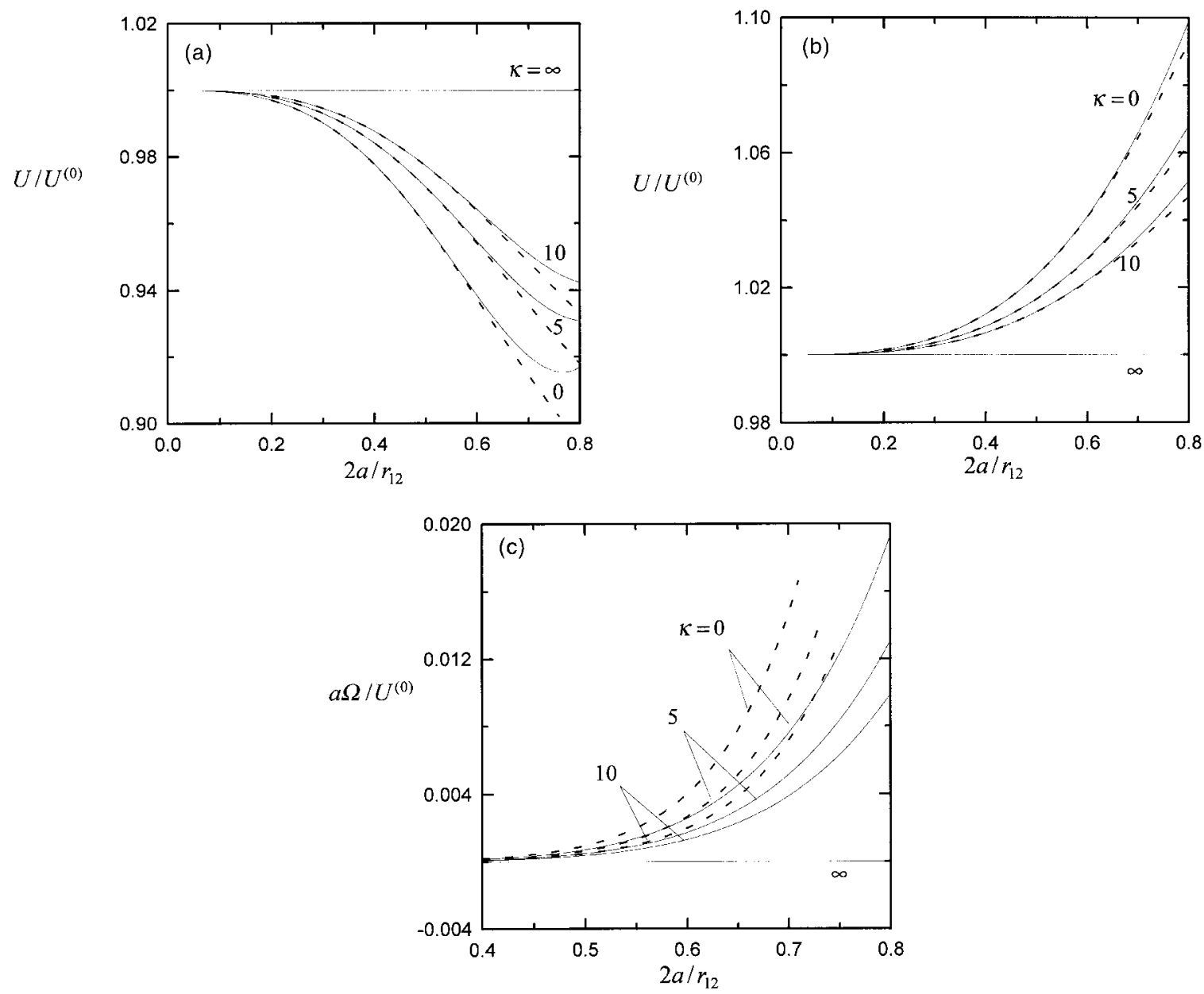

Fig. 2. Normalized translational and rotational velocities of two identical vesicles versus the separation parameter $2 a / r_{12}$ with $\bar{\kappa}=5$ and $\kappa$ as a parameter: (a) solute concentration gradient prescribed parallel to the line of vesicle centers; (b) and (c) solute concentration gradient prescribed perpendicular to the line of vesicle centers. The solid curves are computed from Eqs. (35a) and (35b), while the dashed curves are results obtained using bipolar coordinates.

touching. Interestingly, as shown in Fig. 3(c), a maximum of the angular velocity of vesicle 1 [to the accuracy of $O\left(r_{12}^{-7}\right)$ ] exists for the case of $a_{2} / a_{1}=(\sqrt{43}-4) / 3(\approx 0.85)$ when the parameter $\left(a_{1}+a_{2}\right) / r_{12}$ is kept constant. This behavior, which can also be demonstrated using Eq. (35b), is understandable knowing that the value of $r_{12} / a_{1}$ increases linearly with $a_{2} / a_{1}$ for a fixed $\left(a_{1}+a_{2}\right) / r_{12}$.

In practical applications of osmophoresis, collections of vesicles are usually encountered. It is therefore necessary to determine the dependence of the osmophoretic mobility on vesicle concentration. The interaction effects between pairs of vesicles, obtained in the previous section, can be extended to the evaluation of the average osmophoretic velocity in a suspension 

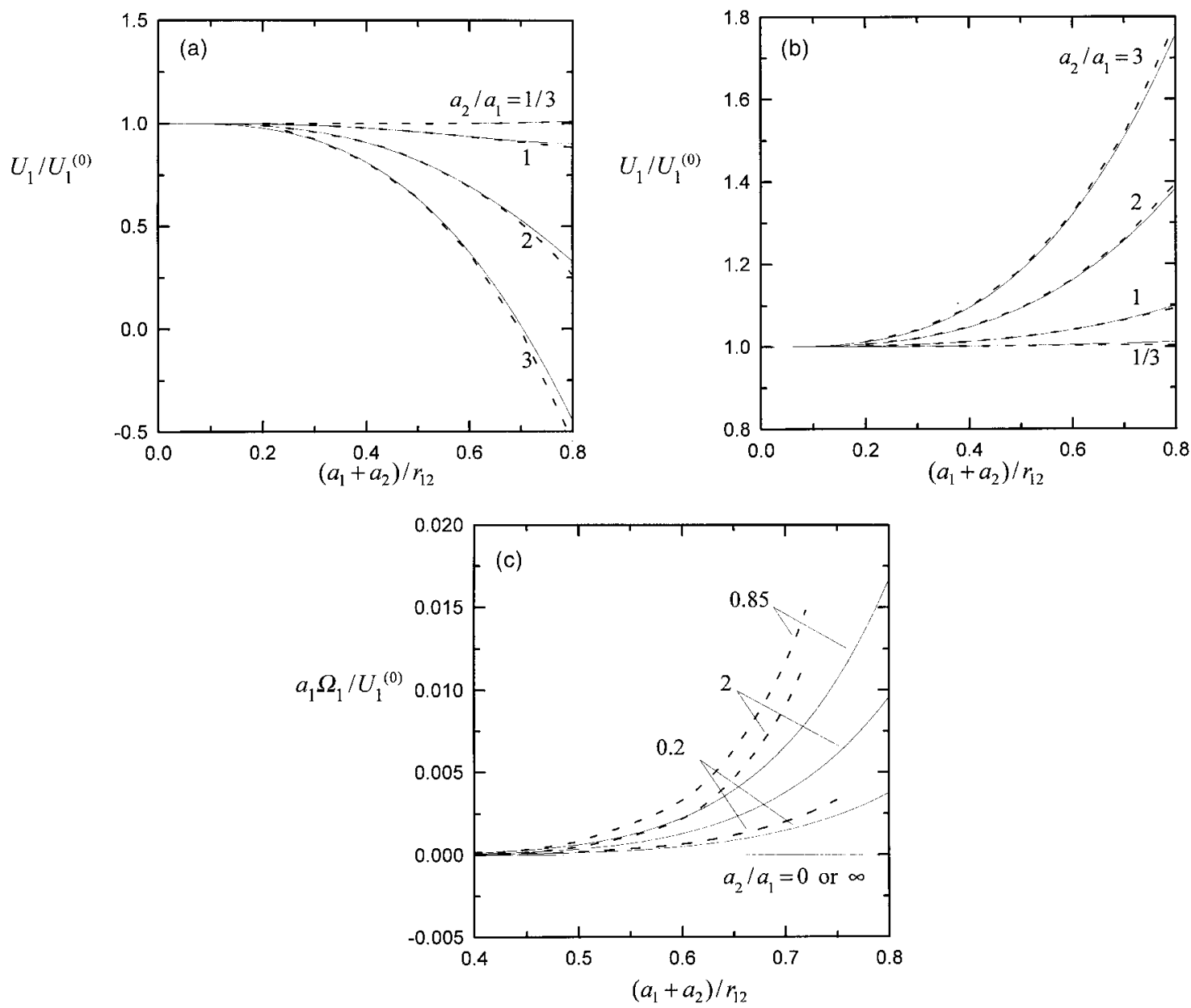

Fig. 3. Normalized translational and rotational velocities of vesicle 1 for the system of two vesicles of identical physical properties with $\kappa=\bar{\kappa}=0$ versus the separation parameter $\left(a_{1}+a_{2}\right) / r_{12}$ with $a_{2} / a_{1}$ as a parameter: (a) solute concentration gradient prescribed parallel to the line of vesicle centers; (b) and (c) solute concentration gradient prescribed perpendicular to the line of vesicle centers. The solid curves are computed from Eqs. (35a) and (35b), while the dashed curves are results obtained using bipolar coordinates.

of spherical vesicles. In the following section, formulas for this average velocity correct to the order of first power of the volume fraction of the vesicles will be presented.

\section{Volume fraction dependence of osmophoretic velocity}

For a bounded suspension of vesicles subjected to an imposed solute concentration gradient $\mathbf{E}_{\infty}$, it is no longer possible to define the vesicle velocity relative to the distant fluid. Instead, 
the vesicle velocity should be calculated for a reference frame in which the net particle and fluid flux is zero and $\mathbf{E}_{\infty}$ is the volume average of the concentration gradient field over the entire suspension. Thus,

$$
\begin{aligned}
& \frac{1}{V} \int_{V} \mathbf{v}(\mathbf{x}) \mathrm{d} \mathbf{x}=\mathbf{0} \\
& \frac{1}{V} \int_{V} \nabla C(\mathbf{x}) \mathrm{d} \mathbf{x}=\mathbf{E}_{\infty}
\end{aligned}
$$

where $V$ denotes the entire volume of the suspension. Due to the existence of vesicles, suspending fluid, and a discontinuity of solute concentration at the surface of each vesicle, Eq. (36b) can be further expressed as

$$
\frac{1}{V}\left\{\int_{V_{\mathrm{s}}} \nabla C(\mathbf{x}) \mathrm{d} \mathbf{x}+\sum_{j}\left[\int_{V_{j}} \nabla \hat{C}_{j}(\mathbf{x}) \mathrm{d} \mathbf{x}+\int_{S_{j}}\left(C(\mathbf{x})-\hat{C}_{j}(\mathbf{x})\right) \mathbf{n}_{j} \mathrm{~d} S\right]\right\}=\mathbf{E}_{\infty}
$$

where $\hat{C}_{j}(\mathbf{x})$ is the solute concentration distribution inside vesicle $j, V_{j}$ and $S_{j}$ are the volume and surface area of vesicle $j$, respectively, $V_{\mathrm{s}}=V-\Sigma_{j} V_{j}$ is the volume occupied by the suspending fluid, and $\mathbf{n}_{j}$ is the unit normal vector at the surface of vesicle $j$ pointing into the surrounding fluid. The surface integral in Eq. (37) accounts for the solute concentration gradient on the surface of vesicle $j$, which is singular.

Based on a microscopic model of particle interactions in a dilute dispersion which involves both statistical and low Reynolds number hydrodynamic concepts (Batchelor, 1972; Reed and Anderson, 1980), the ensemble-averaged osmophoretic velocity of a 'test' vesicle (subscript t) in a suspension of spherical vesicles subject to Eqs. (36a) and (37) can be expressed as

$$
\begin{aligned}
& \left\langle\mathbf{U}_{\mathrm{t}}\right\rangle=\mathbf{U}_{\mathrm{t}}^{(0)}+n\left\{\int_{V} \mathbf{v}^{*}(\mathbf{r})[g(\mathbf{r})-1] \mathrm{d} \mathbf{r}+A_{\mathrm{t}} \int_{V}\left[\mathbf{E}^{*}(\mathbf{r})-\mathbf{E}_{\infty}\right][g(\mathbf{r})-1] \mathrm{d} \mathbf{r}\right. \\
& \left.+A_{\mathrm{t}} \frac{a}{\kappa} \int_{r=a} \frac{\mathbf{r r}}{r^{2}} \cdot \mathbf{E}^{*}(\mathbf{r})[g(\mathbf{r})-1] \mathrm{d} S+\int_{V} \mathbf{W}(\mathbf{r}) g(\mathbf{r}) \mathrm{d} \mathbf{r}\right\}+O\left(n^{2}\right)
\end{aligned}
$$

Here $\mathbf{U}_{\mathrm{t}}^{(0)}=A_{\mathrm{t}} \mathbf{E}_{\infty}$, which is the undisturbed osmophoretic velocity of the test vesicle, $g(\mathbf{r})$ is the radial distribution function describing the two-particle configurational probability, and $n$ is the macroscopic concentration of the neighbor vesicles (assumed to be identical with radius $a$ ). $\mathbf{E}^{*}(\mathbf{r})$ and $\mathbf{v}^{*}(\mathbf{r})$ are the solute concentration gradient and fluid velocity fields, respectively, at position $\mathbf{r}$ when a neighboring vesicle at the origin $\mathbf{0}$ moves due to the prescribed concentration gradient $\mathbf{E}_{\infty}$, which are expressed by Eqs. (31a) and (31b) (eliminating the superscripts and subscripts) for $r \geqslant a$. Inside the neighboring vesicle $(r<a)$, $\mathbf{E}^{*}$ can be obtained using Eq. (11b) and $\mathbf{v}^{*}$ is given by Keh and Yang (1992),

$$
\mathbf{E}^{*}=(1+\hat{\mathbf{G}}) \mathbf{E}_{\infty}
$$




$$
\mathbf{v}^{*}=\left[-5 \mathbf{I}+3\left(\frac{r}{a}\right)^{2}(2 \mathbf{I}-\mathbf{e e})\right] \cdot \mathbf{U}^{(0)}
$$

Note that $\mathbf{E}^{*}$ is constant for $r<a$ and is singular with a discontinuity of solute concentration at $r=a$, while the volume-averaged value of $\mathbf{v}^{*}$ over the fluid inside the neighboring vesicle equals $-2 \mathbf{U}^{(0)}$. W(r) is a correction function needed to account for the perturbation on $\mathbf{v}^{*}$ owing to the presence of the test vesicle, and is given by

$$
\mathbf{W}(\mathbf{r})=\mathbf{U}_{\mathrm{t}}^{*}(\mathbf{r})-\mathbf{U}_{\mathrm{t}}^{(0)}-\mathbf{v}^{*}(\mathbf{r})-A_{\mathrm{t}}\left[\mathbf{E}^{*}(\mathbf{r})-\mathbf{E}_{\infty}\right]
$$

where $\mathbf{U}_{t}^{*}(\mathbf{r})$ is the actual velocity of the test vesicle located at $\mathbf{r}$ with respect to the origin of a single neighboring vesicle at $\mathbf{0}$. $\mathbf{U}_{\mathrm{t}}^{*}(\mathbf{r})$ can be calculated from Eq. (35a), taking subscripts 1 and 2 to denote the test and neighboring vesicles, respectively. Note that the Faxen correction term involving $\nabla^{2} \mathbf{v}^{*}$, which should have appeared in Eqs. (38) and (40), equals zero, as computed from using Eq. (31b).

To evaluate the volume integrals in Eq. (38), we assume that the radial distribution function has the following equilibrium value for rigid spheres without long-range pair potential:

$$
\begin{aligned}
& g=0 \quad \text { if } r<a_{\mathrm{t}}+a \\
& g=1+O(n) \quad \text { if } r>a_{\mathrm{t}}+a
\end{aligned}
$$

where $O(n)$ is a term proportional to the concentration of neighbors. In other words, the vesicles must be sufficiently small so that Brownian motion dominates any multiparticle hydrodynamic interactions that might impart microscopic structure to the suspension. In general, it is necessary to obtain the pair distribution function as the solution of a conservation equation of Fokker-Planck type for a polydisperse system of spheres (Batchelor, 1982). The condition under which the assumption of the local equilibrium is valid for a dilute dispersion consisting of different types of particles are also discussed by Reed and Anderson (1980).

Given Eqs. (31a) and (31b) or (39a) and (39b) for $\mathbf{E}^{*}(\mathbf{r})$ and $\mathbf{v}^{*}(\mathbf{r})$, Eq. (35a) for $\mathbf{U}_{\mathbf{t}}^{*}(\mathbf{r})$, Eq. (40) for $\mathbf{W}(\mathbf{r})$, and Eq. (41) for $g(\mathbf{r})$, the integrals in Eq. (38) are evaluated to obtain

$$
\left\langle\mathbf{U}_{\mathrm{t}}\right\rangle=\mathbf{U}_{\mathrm{t}}^{(0)}\left[1+\alpha_{\mathrm{t}} \varphi+O\left(\varphi^{2}\right)\right]
$$

with

$$
\alpha_{\mathrm{t}}=2 \frac{A}{A_{\mathrm{t}}}-\hat{G}-(G-\hat{G})+\left[2 G_{\mathrm{t}} G+2 G_{\mathrm{t}} \frac{A}{A_{\mathrm{t}}}-2 G_{\mathrm{t}} \frac{B}{A_{\mathrm{t}}}+5\right]\left(\frac{a_{\mathrm{t}}}{a_{\mathrm{t}}+a}\right)^{3}
$$

where $\varphi=4 \pi \mathrm{a}^{3} n / 3$ is the volume fraction of the neighbor vesicles. The four terms in the expression (43) for $\alpha_{t}$ are obtained in order of the contributions from the first, second, third, and fourth integrals in Eq. (38). This result is not exact, even given Eqs. (41a) and (41b) hold, because $O\left(r_{12}^{-8}\right)$ terms are neglected in $\mathbf{U}_{\mathrm{t}}^{*}(\mathbf{r})$; however, the error should be small and will appear only in the calculation involving the correction function $\mathbf{W}(\mathbf{r})$. In the derivation of Eq. (43), all the neighbor vesicles were assumed to be identical, even though they are allowed to differ from the test vesicle. 
For a dispersion of vesicles that have a distribution in radius and physical properties, a generalization of Eqs. (42) and (43) yields

$$
\begin{aligned}
& \left\langle\mathbf{U}_{i}\right\rangle=\mathbf{U}_{i}^{(0)}\left[1+\sum_{j} \alpha_{i j} \varphi_{j}+O\left(\varphi^{2}\right)\right] \\
& \alpha_{i j}=2 \frac{A_{j}}{A_{i}}-G_{j}+\left[2 G_{i} G_{j}+2 G_{i} \frac{A_{j}}{A_{i}}-2 G_{i} \frac{B_{j}}{A_{i}}+5\right]\left(\frac{a_{i}}{a_{i}+a_{j}}\right)^{3}
\end{aligned}
$$

Here, the subscript $i$ denotes the type of vesicles having radius $a_{i}$ and properties $\kappa_{i}$ and $\bar{\kappa}_{i}$.

In a suspension of identical vesicles, the expression for the average osmophoretic velocity can be reduced from Eqs. (44) and (45) to

$$
\begin{aligned}
& \langle U\rangle=\mathbf{U}^{(0)}\left[1+\alpha \varphi+O\left(\varphi^{2}\right)\right] \\
& \alpha=2-G+\frac{1}{8}\left[2 G^{2}+2\left(1-\frac{B}{A}\right) G+5\right]
\end{aligned}
$$

For the limiting situation of $\kappa \rightarrow \infty(\mathrm{G}=1 / 2$ and $B / A=-5 / 2)$, we have $\alpha=3$ and the contribution from the correction function $\mathbf{W}$ to $\alpha$ vanishes. Note that $\alpha$ is nonzero in this limit, even though there is no interaction between two vesicles in an unbounded fluid. The reason for this is that in the bounded suspension the volume-averaged flow is zero (this contributes 2 to $\alpha$ ) and the volume-averaged concentration gradient is $\mathbf{E}_{\infty}$ (this contributes 1 to $\alpha$ ) as required by Eq. (36). For the limiting case of $\bar{\kappa} \rightarrow \infty$, Eqs. (10a), (22) and (26) give $G=1 / 2$ and $B /$ $A=-10 / 3$; then Eq. (47) yields $\alpha=131 / 48$. On the other hand, for the limiting situation of $\kappa=\bar{\kappa}=0$, one has $G=1 / 2$ and $B / A=-5 / 3$; then Eq. (47) gives $\alpha=121 / 48$, which was obtained by Anderson (1986).

Results of $\alpha$ calculated from Eq. (47) for a suspension of identical vesicles at various values of $\kappa$ and $\bar{\kappa}$ are shown in Fig. 4. It can be found in all cases that the average osmophoretic velocity always increases with an increase in the vesicle concentration (with $121 / 48 \leqslant \alpha \leqslant 3$ ). This behavior, which is contrary to that for sedimentation (Batchelor, 1972), electrophoresis/ diffusiophoresis (Anderson, 1986) and thermocapillary motion (Anderson, 1985), is understandable because the direction of the solvent flow is opposite to that of the vesicle's movement and the back flow of solvent in the bounded suspension enhances the migration of vesicles. Although the value of $\alpha$ in general increases with the increase of $\kappa$, as exhibited in Fig 4(a), the dependence is not necessarily monotonic. Note that the effect of interactions between two vesicles (in an unbounded fluid) is to reduce the value of $\alpha$.

\section{Acknowledgements}

This research was supported by the National Science Council of the Republic of China under grant NSC88-2214-E-002-010. 

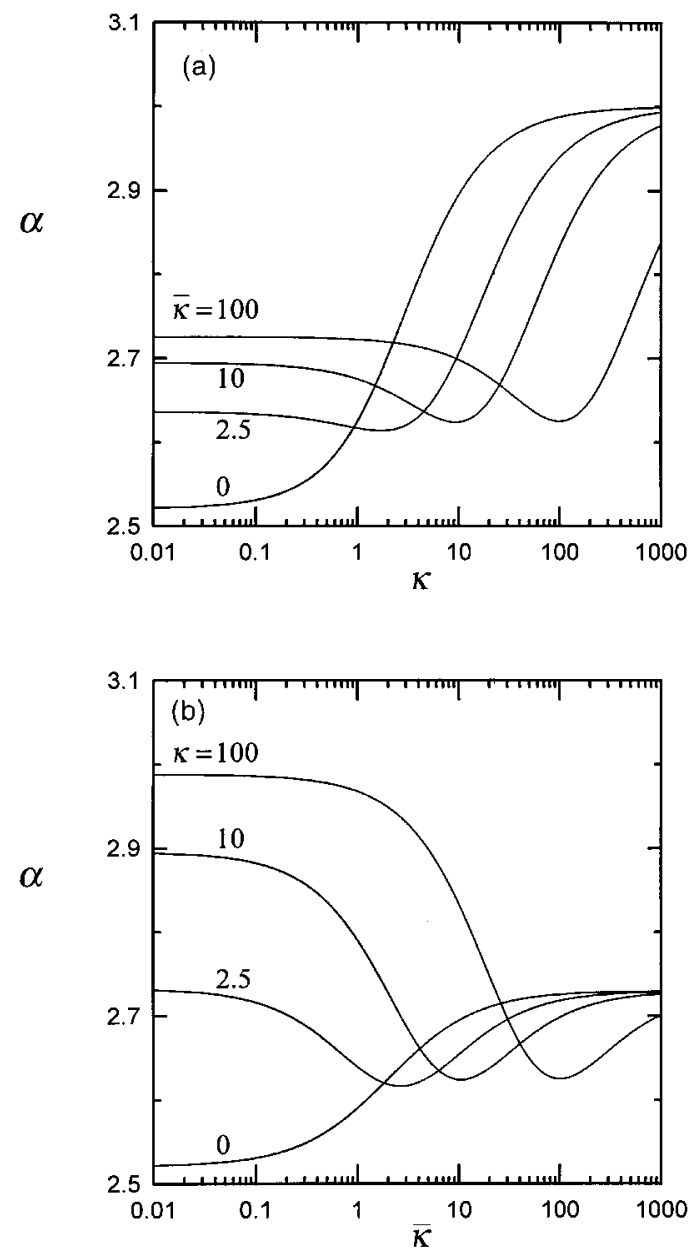

Fig. 4. Coefficient $\alpha$ calculated from Eq. (47) for a suspension of identical vesicles: (a) $\alpha$ versus $\kappa$ with $\bar{\kappa}$ as a parameter; (b) $\alpha$ versus $\bar{\kappa}$ with $\kappa$ as a parameter.

\section{References}

Anderson, J.L., 1983. Movement of a semipermeable vesicle through an osmotic gradient. Phys. Fluids 26, 28712879.

Anderson, J.L., 1984. Shape and permeability effects on osmophoresis. PhysicoChem. Hydrodyn. 5, $205-216$.

Anderson, J.L., 1985. Droplet interactions in thermocapillary motion. Int. J. Multiphase Flow 11, 813-824.

Anderson, J.L., 1986. Transport mechanisms of biological colloids. Ann. NY Acad. Sci. (Biochem. Engng IV) 469, 166-177.

Barton, J.W., Ford, R.M., 1997. Mathematical model for characterization of bacterial migration through sand cores. Biotechnol. Bioengng 53, 487-496.

Batchelor, G.K., 1972. Sedimentation in a dilute dispersion of spheres. J. Fluid Mech. 52, 245-268.

Batchelor, G.K., 1982. Sedimentation in a dilute polydisperse system of interacting spheres. Part 1. General theory. J. Fluid Mech. 119, 379-408. 
Brenner, H., 1964. The Stokes resistance of a slightly deformed sphere. Chem. Engng Sci. 19, 519-539.

Brenner, M.P., Levitov, L.S., Budrene, E.O., 1998. Physical mechanisms for chemotactic pattern formation by bacteria. Biophys. J. 74, 1677-1693.

Chen, S.B., Keh, H.J., 1988. Electrophoresis in a dilute dispersion of colloidal spheres. AIChE J 34, $1075-1085$.

Devreotes, P.N., Zigmond, S.H., 1988. Chemotaxis in enkaryotic cells: a focus on leukocytes and dictyostelium. A. Rev. Cell Biol. 4, 649-686.

Gordon, L.G.M., 1981. Osmophoresis. J. Phys. Chem. 85, 1753-1755.

Happel, J., Brenner, H., 1983. Low Reynolds Number Hydrodynamics. Nijhoff, The Hague, The Netherlands.

Keh, H.J., Yang, F.R., 1992. Particle interactions in osmophoresis. Int. J. Multiphase Flow 18, 593-615.

Reed, C.C., Anderson, J.L., 1980. Hindered settling of a suspension at low Reynolds number. AIChE J. 26, 816827. 